\title{
Social Comparison and Its Relation to Body Dissatisfaction in Bulimia Nervosa: Evidence From Eye Movements
}

\author{
Jens Blechert, PhD, Till Nickert, Dipl Psych, Detlef Caffier, Dipl Mat, and Brunna Tuschen-Caffier, PhD
}

\begin{abstract}
Objective: To determine the role of social comparison for body dissatisfaction in bulimia nervosa (BN). Previous research suggested that exposure to media content idealizing thin body shape decreases body satisfaction, particularly in women with eating disorder symptoms. This might be due to the negative outcome of social comparisons with media models, and women with eating disorders might be particularly susceptible because they engage in upward social comparison more frequently than women without these symptoms. However, no study has yet explored both upward (i.e., toward more attractive others) and downward (i.e., toward less attractive others) social comparisons and their impact on body dissatisfaction in a clinical eating disorder and healthy controls. Methods: We presented patients with BN $(n=20)$ and healthy controls $(\mathrm{HC})(n=22)$ with slides comprising a digitized image of their own body alongside comparison bodies with higher and lower body mass indices (BMIs) while measuring their eye movements. Results: As hypothesized, patients with BN fixated longer on comparison bodies with lower BMIs than controls, with the reverse pattern for high BMI bodies. This gaze pattern suggests that upward comparisons were more prevalent in the BN group. Furthermore, upward comparisons were related to a drop in body satisfaction in the BN group. Conclusions: Disadvantageous social comparison strategies might be related to body dissatisfaction and therefore to the maintenance of BN. Key words: bulimia nervosa, eye movements, eye tracking, social comparison, body image disturbance, body dissatisfaction.
\end{abstract}

BISS = Body Image State Scale; BMI = body mass index; $\mathbf{B N}=$ bulimia nervosa; DSM-IV = Diagnostic and Statistical Manual of Mental Disorders, 4th Edition; EDE-Q = Eating Disorder Examination Questionnaire; HC = healthy controls.

\section{INTRODUCTION}

$B^{\text {ol }}$ dy image dissatisfaction has received the greatest empirical support as a precursor to eating disturbances (1-3) and is widespread among women in Western societies $(4,5)$. According to sociocultural theory, current societal standards for beauty propagate a thin ideal that many women are unable to achieve (2). Research has started to explore how this hardly attainable but nevertheless idealized thin ideal is transmitted and internalized. Numerous studies have investigated the impact of the thin ideal in the mass media on body dissatisfaction, using correlational and experimental designs (6). Results consistently find a positive correlation between media exposure and body dissatisfaction, but the exact mediation and direction of causality are not yet fully understood. Moreover, although the pervasiveness of the media means that almost all women are confronted with idealized thin beauty images, only a minority develop clinically relevant body shape and eating concerns. Given this, certain mechanisms may be involved in the translation of norms promoted by the media into individual body dissatisfaction which represents an important aspect of eating disorder symptomatology.

Among a number of potential mediating mechanisms, social comparison seems to play a pivotal role linking media consumption and body dissatisfaction (6-8) and individuals with eating disorder symptoms engage in more frequent and more disadvantageous social comparisons than asymptomatic

From the Department of Clinical Psychology and Psychotherapy, Institute for Psychology, University of Freiburg, Freiburg, Germany.

Address correspondence and reprint requests to Jens Blechert, Department of Clinical Psychology and Psychotherapy, Institute for Psychology, University of Freiburg, Engelbergerstrasse 41, 79106 Freiburg, Germany. E-mail: Blechert@psychologie.uni-freiburg.de

Received for publication January 20, 2009; revision received April 29, 2009.

This research was supported, in part, by Grant TU78-6-1 from the German Research Foundation (B.T.-C.).

DOI: $10.1097 /$ PSY.0b013e3181b4434d controls (9-11). Social comparison theory postulates a need for self-evaluation, which, in the absence of absolute comparison norms, can best be satisfied by a comparison to relevant others. Upward comparisons are comparisons to superior peers and are thought to be adopted when self-improvement is intended. Failure in self-improvement, however, can threaten self-esteem. Downward comparisons, by contrast, are thought to serve the protection of self-worth (12). Stable interindividual differences in the tendency to engage in social comparisons have been found (13). However, social comparison can also be triggered by certain experimental situations or instructions (8).

Social comparison research related to body satisfaction has thus far utilized mostly media images or videos of thin models. This focus has three limitations. First, because models in the media are almost always slimmer and perceived as more attractive than oneself, social comparisons with these models are necessarily upward and therefore potentially deleterious for self-esteem and body satisfaction. The possibly advantageous effects of downward comparisons (i.e., to less attractive others) remained unexplored in previous research. Second, the social environment contains not only highly idealized media images, but a range of possible targets for social comparisons. Thus, in everyday life, individuals have a choice of whom to compare themselves with. This fact is not reflected in previous studies, using standardized exposure to media models. Third, and related to this, social comparisons are more likely to be made to relevant others, that is, individuals perceived to belong to the same category like oneself (14). Thus, popular media models are probably less relevant for social comparisons than peers $(9,15)$.

A further limitation of previous research is the reliance on self-report measures, which are characterized by several wellknown problems. To remedy this, we used eye-movement registration as our main dependent variable. Eye movements closely follow shifts in visual attention (16). Although in principle under voluntary control, eye movements are typically not monitored explicitly (17) and may thus yield valuable insights into more implicit social comparison strategies than accessible by subjective report. Studies using this meth- 
odology indicate that body dissatisfaction is closely related to visual gaze patterns on the own body and on comparison bodies (18, Blechert, Ansorge, Tuschen-Caffier, submitted).

To extend and clarify previous research, the present study investigated whether the selection of a comparison strategy (upward focus on more attractive others versus downward focus on less attractive others) differentiates patients with bulimia nervosa $(\mathrm{BN})$ and healthy controls $(\mathrm{HC})$, and whether it is a significant determinant of body dissatisfaction. Furthermore, because peers are possibly more relevant for social comparisons than media models, we used standardized pictures of headless bodies of unselected women as comparison targets. Participants viewed slides comprising the participants' own body in the middle flanked by two comparison bodies with higher and lower body mass indices (BMIs) (ratio of weight to squared height in $\mathrm{kg} / \mathrm{m}^{2}$ ) on the left and right sides. Bias toward upward or downward comparisons was inferred from viewing times of the bodies with higher/lower BMI, as measured by continuous eye movement registration. We expected $\mathrm{BN}$ patients to engage in more upward comparison, that is, longer fixation times for bodies with lower BMIs, compared with controls. Furthermore, body satisfaction, measured before and after picture viewing, was expected to decrease in the BN group but not in the control group.

\section{METHODS}

\section{Participants}

The study sample consisted of 20 women diagnosed with BN and 22 women with noneating disorders for the healthy control group (HC). Ethical approval for the conduct of this study was granted by the German Psychological Society's ethics committee. Participants took part in exchange of a remuneration of $€ 50$ and were recruited from the community through newspaper announcements, the department's Web site, and from collaborating clinics. All participants had normal or corrected-to-normal vision.

Exclusion criteria for all participants were schizophrenia spectrum disorders, bipolar disorder, substance abuse or dependence, or neurological disorders. Exclusion criteria for $\mathrm{HC}$ participants included a lifetime diagnosis of any mental disorder according to the Diagnostic and Statistical Manual of Mental Disorders, 4th Edition (DSM-IV). The German versions of the Eating Disorder Examination (EDE) (19) and the Structured Clinical Interview for DSM-IV (20) were used for the diagnosis of BN and all other psychiatric diagnoses, respectively. The following comorbid disorders were found in the BN group: major depression (6), borderline personality disorder (2), posttraumatic stress disorder (1), and social phobia (1). The data collection period extended from October 2007 to December 2008.

\section{Psychometric Measures}

Eating disorder psychopathology and body image disturbance were assessed with the German versions of the Eating Disorder Examination-Questionnaire (21), the Body Image Avoidance Questionnaire (22), and the Body Checking Questionnaires (23). Habitual frequency of social comparisons were assessed with the German version of the Physical Appearance Comparison Scale (24). An example item of the Physical Appearance Comparison Scale is "At parties or other social events, I compare my physical appearance to the physical appearance of others." The Rosenberg Self-Esteem Scale (25) measured self-esteem. Good internal consistency and test-retest reliability have been demonstrated for all of these scales (21-25).

Body dissatisfaction was measured before and after the picture viewing, using the Body Image State Scale, BISS $(26,27)$. Using six items, this scale assesses various aspects of body image, such as dissatisfaction with one's overall physical appearance, size, shape and weight, feelings of physical
TABLE 1. Mean (Standard Deviation) Values and Results of $t$ Tests for Sample Characteristics

\begin{tabular}{|c|c|c|c|}
\hline & Bulimia N. & $\begin{array}{l}\text { Healthy } \\
\text { Controls }\end{array}$ & $t, p$ \\
\hline Number in sample & 20 & 22 & \\
\hline Age, years & $26.6(7.68)$ & $26.5(4.65)$ & $0.01, .939$ \\
\hline Years of education & $11.7 \quad(1.83)$ & $12.8(0.64)$ & $7.18, .011$ \\
\hline $\begin{array}{l}\text { Body mass index, } \\
\mathrm{kg} / \mathrm{m}^{2}\end{array}$ & $22.6(3.40)$ & $20.3(2.24)$ & $6.70, .013$ \\
\hline $\begin{array}{l}\text { Beck Depression } \\
\text { Inventory }\end{array}$ & 18.1 (8.49) & $2.81(3.09)$ & $60.0,<.001$ \\
\hline EDE-Q restrained & $3.27(1.92)$ & $0.47(0.81)$ & $37.4,<.001$ \\
\hline EDE-Q eating concerns & $3.73(1.32)$ & $0.13(0.26)$ & $150,<.001$ \\
\hline EDE-Q weight concerns & $4.17(1.46)$ & $0.44(0.56)$ & $117,<.001$ \\
\hline EDE-Q shape concerns & $4.75(0.97)$ & $0.67(0.75)$ & $225,<.001$ \\
\hline $\begin{array}{l}\text { Body Image Avoidance } \\
\text { Quest. }\end{array}$ & $17.4 \quad(8.02)$ & $5.36(3.93)$ & $39.2,<.001$ \\
\hline $\begin{array}{l}\text { Body Checking } \\
\text { Questionnaire }\end{array}$ & $1.76(0.72)$ & $0.52(0.21)$ & $60.8,<.001$ \\
\hline $\begin{array}{l}\text { Rosenberg Self-Esteem } \\
\text { Scale }\end{array}$ & $1.99(0.55)$ & $2.81(0.51)$ & $25.7,<.001$ \\
\hline $\begin{array}{l}\text { Physical Appearance Social } \\
\text { Comparison Scale }\end{array}$ & $3.98(0.94)$ & $2.48(0.64)$ & $31.1,<.001$ \\
\hline
\end{tabular}

Bulimia $\mathrm{N}$ = bulimia nervosa; EDE-Q = Eating Disorder Examination Questionnaire.

unattractiveness, concerns about how one looks at the present moment relative to how one usually looks, and evaluation of one's own appearance relative to how the average person looks. The BISS has proven to be acceptably internally consistent in multiple contexts (Cronbach's $\alpha=0.77-0.90$ ) and moderately stable (test-retest reliability after $2-3$ weeks: $0.69 ; 26$ ).

As indicated in Table 1, groups did not differ in age, but patients with BN had lower education and higher BMI. Thus, BMI was included as a covariate in all analyses. Preliminary analyses indicated that education as a covariate was not significant in any analysis, all $F$ values $<1.00$. As could be expected, patients with $\mathrm{BN}$ had higher eating disorder psychopathology as indicated by the subscales of the Eating Disorder Examination-Questionnaire, Body Image Avoidance Questionnaire, and Body Checking Questionnaires scales, compared with HC. Further, patients with BN had lower self-esteem and engaged more frequently in social comparison.

\section{Materials and Procedure}

After a telephone screening, eligible participants were invited to a diagnostic session during which the Eating Disorder Examination and Structured Clinical Interview for DSM-IV interviews were conducted and the photographs were taken. They were asked to put on a beige leotard and to stand in front of a black background. Digital pictures were taken by a female experimenter from the front, side, and back views, excluding the head. Thereafter, the participant's weight, height, waist and hip circumference were determined and BMI was calculated.

Three comparison bodies with higher BMIs (participant's BMI +2 to +4 BMI points) and three comparison bodies with lower BMIs (participant's BMI -2 to -4 BMI points) were located $8.5^{\circ}$ of visual angle left and right of the participant's body in one compound slide. Body pictures were gray scale and matched in size, brightness, and contrast. Each of six comparison bodies appeared once on the left and once on the right side from each view (front, side, back). Thus, 36 pictures were generated ( 6 comparison bodies $\times$ 2 sides $\times 3$ perspectives).

Participants returned for the experimental session approximately $1 \mathrm{wk}$ later. Participants first completed the BISS questionnaire. Then, after the fitting of the eye-tracking gear and a 10-min peripheral cueing procedure which involved repeated presentations of their own picture (Blechert, Ansorge, Tuschen-Caffier, submitted), the present investigation commenced 
with written instructions: "In the following pictures you will see yourself in the middle and several other persons to your left and right hand side. Just look at the pictures, you will be asked to evaluate them later." Each picture was shown for 6 seconds, followed by a 1.5 -second intertrial interval during which a fixation cross was displayed on which participants were to direct their gaze. No button presses or other responses were required. Three practice trials preceded the 36 experimental trials.

Subsequent to the picture viewing, participants rated their own body and the six comparison bodies. The bodies were presented on the screen in front, side, and back views along with a 20-point visual analog scale on which a slider could be moved from a zero midpoint by clicking the left or right mouse button. Ratings of attractiveness (anchors: "attractive" and "unattractive") and body shape (anchors: "thin" and "fat") were obtained for each body and view ( 7 bodies $\times 3$ views $\times 2$ scales). Then, the BISS questionnaire was applied a second time.

\section{Apparatus, Data Acquisition, and Data Reduction}

Testing took place in a sound-attenuated windowless chamber partitioned into a testing and an experimenter room. The experimenter could communicate with participants by intercom and observe them through an unobtrusive video camera. One Pentium 3 PC ran Presentation (Neurobehavioral Systems, Albany, California), which controlled the picture presentation and communicated with a second PC controlling the table-mounted, height-adjustable 240-Hz Eyelink eyetracker (Sensomotoric Instruments, Berlin, Germany). Picture presentation and real-time eye movements were mirrored by two monitors in the experimenter room, allowing for a careful monitoring of the data quality and execution of procedures. Pictures were presented on a 17-inch monitor at $100 \mathrm{~Hz}$ at 80 -cm viewing distance. A 5-point calibration procedure preceded the picture presentation.

Off-line, fixation time was calculated as the overall time gaze coordinates lay on either side of the composite slide (center $\pm 3.3^{\circ}$ ), separately for each side (left/right) and comparison body (high BMI side/low BMI side). We also analyzed saccade latency (calculated as the time of picture presentation to the first horizontal shift of $>3.3^{\circ}$ from the center) and the direction of the first saccade but found no group differences on these parameters. Blink times of blinks that occurred at the time fixation was on one of the comparison bodies were included into the respective fixation time while times of erroneous eye-tracking data were excluded. Preliminary analyses indicated no group differences in blink times or proportion of erroneous data. Furthermore, no group differences were found in fixation times with respect to sides (right, left) or body views (front, side, back). Thus, eye movement and rating data were collapsed across all 36 pictures. To obtain comprehensive scores, fixation times were converted into percentages: time_high $\mathrm{BMI}_{\mathrm{BM}}$ and time_ low $_{\mathrm{BMI}}$ expressed fixation times on comparison bodies with higher/lower BMI as percentages of the total presentation time $(6 \mathrm{~s} \times 36)$. As a control measure, time_self was calculated as total time - the sum of time_high ${ }_{\text {BMI }}$

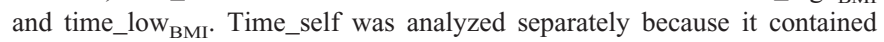
data from several sources: 1) latency of the first saccade (participants fixated on the middle position during the intertrial interval);2) saccadic shifts across the midline; and 3) periods of invalid eye tracking data. Further, the selfpicture cannot be directly compared with the lateral pictures because of different degrees of novelty: Whereas the lateral pictures varied (six different bodies), there was only one self-picture.

Statistical analysis was carried out with SPSS (Version 15; SPSS Inc., Chicago, Illinois). The $\alpha$ level was set to 0.05 . Effect sizes are reported (partial $\eta^{2}, \%$ ). BMI differed across groups and was therefore included as a covariate in all analyses. All statistical tests were two-tailed.

\section{RESULTS}

\section{Fixation Time}

Two healthy participants had to be excluded from this analysis due to insufficient quality of the eye movement data. An initial $2 \times 3 \times 2 \times 2$, Side (right, left) $\times$ View (front, side, back) $\times$ Body (high BMI, low BMI) $\times$ Group $(\mathrm{BN}, \mathrm{HC})$ analysis of covariance (ANCOVA) with Side, View, and Body as repeated measures factors, Group as between-participant factor, and BMI

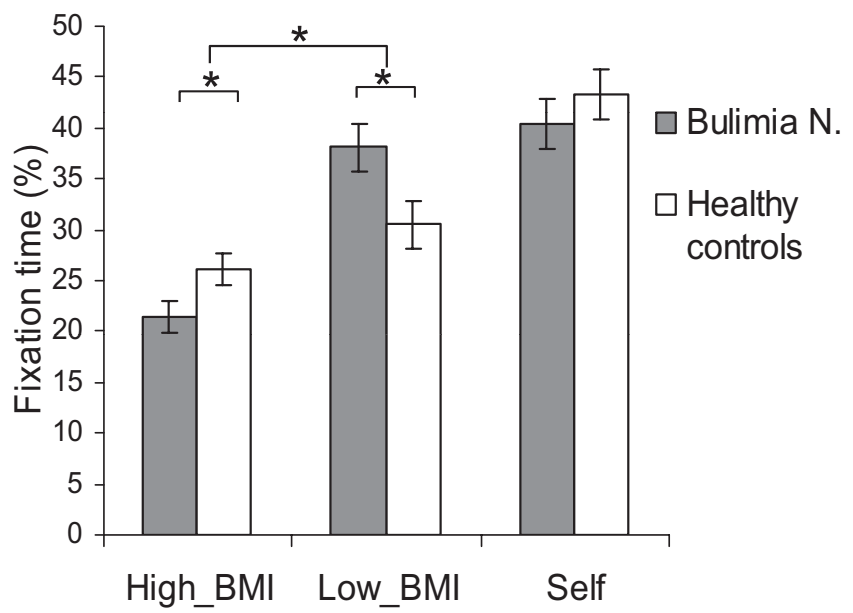

Figure 1. Fixation time (\%) for own body (Self) and comparison bodies with higher and lower body mass index (BMI) (mean, standard error). Bulimia $N$, bulimia nervosa.

as covariate indicated that Side and View did not show any main effects of interactions with Group, $F<1.00$. Thus, fixation times were collapsed across Side and View and submitted to a $2 \times 2$, Body (high BMI, low BMI) $\times$ Group $(\mathrm{BN}, \mathrm{HC})$ ANCOVA, which yielded a highly significant Group $\times$ Body interaction, $F(1,37)=7.66, p=.009, \eta^{2}=17.1 \%$, but no main effects of Group or Body, $p>.167$. BMI was significant, $F(1,37)=5.36$, $p=.026$, partial $\eta^{2}=17.0 \%$ but did not interact with Body, $F<$ 1.00. Follow-up univariate ANCOVAs confirmed this pattern: patients with BN fixated longer on bodies with lower BMIs than controls, $F(1,37)=5.00, p=.032$, with the reverse pattern for high BMI bodies, $F(1,37)=4.55, p=.040$ (see Figure 1). Because time_self might be less reliable than time_high BMI $_{\text {B }}$ and time_low ${ }_{\text {BMI }}$, it was analyzed separately. No Group differences were found for participants' own (Self) pictures, $F<1.00$.

\section{Picture Ratings}

Figure 2 displays verbal ratings for attractiveness (a) and body shape (b) for the participants' own body and the comparison bodies. Attractiveness ratings were subjected to an ANCOVA containing the between-subjects factor Group (BN, HC) and the within-subject factor Body $\left(\operatorname{high}_{\mathrm{BMI}}\right.$, self, low $\left._{\mathrm{BMI}}\right)$. This analysis yielded a significant Group $\times$ Body interaction, $F(2,78)=17.7$, $p<.001$, partial $\eta^{2}=31.3 \%$ in addition to a Group main effect, $F(1,39)=25.9, p<.001, \eta^{2}=40.0 \%$ (BMI: $F<1.00$ ). As indicated by follow-up comparisons, patients with BN gave similar attractiveness ratings for the bodies with lower BMIs, $F(1,39)=1.24, p=.271$, but lower attractiveness ratings for their own body and the bodies with higher BMI than controls, $F(1,39)=45.1, p<.001, \eta^{2}=53.7 \%$ and $F(1,39)=10.4, p=$ $.003, \eta^{2}=21.1 \%$, respectively.

A similar pattern emerged for body shape ratings. An initial Group $\times$ Body ANCOVA revealed a significant Group $\times$ Body interaction, $F(2,78)=7.83, p=.002, \eta^{2}=17.1 \%$ in addition to a Group main effect, $F(1,39)=17.9, p<.001$, $\eta^{2}=32.1 \%$. The covariate BMI was significant, $F(1,39)=$ $8.69, p=.005, \eta^{2}=18.6 \%$, but did not interact with Body, 

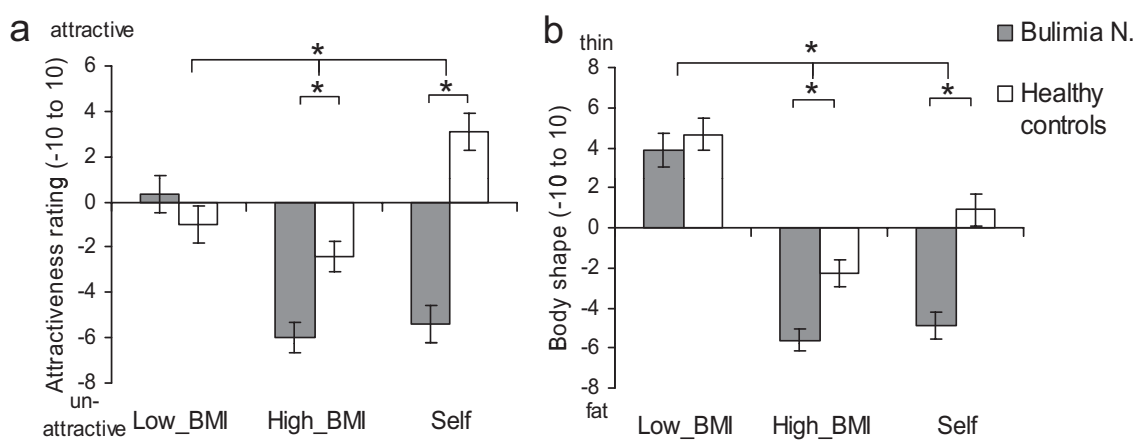

Figure 2. Verbal ratings on attractiveness (a) and body shape (b) for own picture (Self) and comparison bodies with higher and lower body mass index (BMI) (mean, standard error). Bulimia $N$, bulimia nervosa.

$F<1.00$. Patients with BN gave similar body shape ratings for the bodies with lower BMIs, $F(1,38)<1.00$, but perceived their own body shape and the shape of bodies with higher BMIs as larger than did controls, $F(1,39)=31.8, p<.001$, $\eta^{2}=44.9 \%$ and $F(1,39)=11.8, p<.001, \eta^{2}=23.7 \%$, respectively.

\section{Changes on the Body Image State Scale (BISS)}

To assess whether the exposure to comparison bodies influenced differentially body satisfaction, the BISS scores measured before and after picture viewing were analyzed. Preliminary analyses revealed strong group difference on the BISS before picture viewing (mean $[\mathrm{M}], \mathrm{SD}[\mathrm{SD}]$ in the $\mathrm{BN}$ and $\mathrm{HC}$ group: $\mathrm{M}=3.53, \mathrm{SD}=1.70 ; \mathrm{M}=6.72, \mathrm{SD}=1.13$, respectively. Following recommendations of Jamieson (28), simple change scores were computed $(\triangle \mathrm{BISS}=\mathrm{BISSprä} \mathrm{-}$ BISSpost) and submitted to a univariate ANCOVA containing Group (BN, HC) and BMI as covariate, after deletion of one outlier in the HC group ( $\triangle$ BISS z score $>3$ ). A significant Group effect, $F(1,38)=5.12, p=.030, \eta^{2}=11.9 \%$ pointed to a decline of BISS scores in the BN group (adjusted mean$\left[\operatorname{mean}_{\mathrm{adj}}\right]=0.54$, standard error $=0.28$ ) but an increase in the $\mathrm{HC}$ group $\left(\right.$ mean $_{\mathrm{adj}}=-0.27$, standard error $\left.=0.27\right)$. The covariate BMI was not significant, $F<1.00$.

\section{Correlational Analysis}

To determine whether fixation times for high and low BMI comparison bodies were related to attractiveness ratings, or body shape ratings, a bias score for fixation time was calculated (time_low BMI $_{\text {BM }}$ - time_high BMI $_{\text {BM }}$ ) and within-group correlations were computed. As indicated in Table 2, correlations in the $\mathrm{HC}$ group were generally not significant. Within the BN group, the more attractive the $\mathrm{BN}$ patients rated the bodies with lower BMIs, the stronger their fixation bias toward them, whereas the opposite pattern emerged for the bodies with higher BMIs. Body shape ratings showed a similar pattern for bodies with higher BMIs. Thus, the bias toward bodies with low BMIs was related to the perceived attractiveness of these bodies, although this relationship was less reliable in the HC group. Correlations with ratings for the participants' own body indicated that the less attractive and the larger patients with BN perceived themselves, the more they displayed a bias toward bodies with lower BMIs.
TABLE 2. Correlations of Social Comparison Strategy (Fixation Time_Low $_{\text {BMI }}$ - Fixation Time_High Each Group, After Adjustment for BMI

\begin{tabular}{ccc}
\hline & BN r(20) & HC r(22) \\
\hline Low $_{\text {BMI }}$ & & \\
Attractive & $0.509^{a}$ & -0.122 \\
Body shape & 0.099 & -0.321 \\
High & & \\
Attractive & $-0.444^{a}$ & -0.332 \\
Body shape & $-0.474^{a}$ & -0.143 \\
Self & & -0.145 \\
Attractive & $-0.551^{a}$ & -0.158 \\
Body shape & $-0.455^{a}$ & \\
\hline
\end{tabular}

${ }^{a} \alpha<0.05$.

$\mathrm{BMI}=$ body mass index; $\mathrm{BN}=$ bulimia nervosa; $\mathrm{HC}=$ healthy controls.

\section{DISCUSSION}

To our knowledge, this is the first investigation of social comparison processes in bulimic patients and $\mathrm{HC}$, using eyetracking methodology. We addressed several limitations of previous research: In our study, stimulus materials comprised peers instead of models, and allowed for both upward and downward comparisons. Importantly, assessment of fixation times circumvented the limitations of subjective report.

Whereas HC participants showed comparable fixation times for bodies with higher and lower BMIs, patients with BN showed a clear preference for pictures with lower BMIs. This selective attentional pattern can be interpreted as representing social comparison strategies: patients with BN engaged in more upward social comparison and less downward comparison than controls. Importantly, as hypothesized, this strategy seemed to affect their body satisfaction, measured before and after picture viewing: Body satisfaction decreased in patients with $\mathrm{BN}$ but increased in HC. Within-group correlations suggested that the preference for bodies with lower BMIs was related to the perceived attractiveness of these bodies in patients with $\mathrm{BN}$ whereas no such association was present in HC. Furthermore, dissatisfaction of BN patients with their own picture was related to their preference for upward comparisons. Finally, in line with previous research $(9,10)$, the Physical Appearance Comparison Scale scores indicate a habitual tendency of patients with $\mathrm{BN}$ to engage in social comparisons more often than controls. 
How could the preference for upward social comparisons in the BN group be explained? The social comparison literature has related comparison strategies (up- or downward) to the underlying motives of self-enhancement and self-protection $(12,14)$. Applied to our results, this would suggest that patients with BN seek self-enhancement by preferring upward comparisons. This is in contrast to the drop in body satisfaction in this group, and hence, an important aspect of self-evaluation. Furthermore, because the patients with $\mathrm{BN}$ also had lower self-esteem, this finding is in contrast with results of Wood and colleagues indicating that healthy individuals with low self-esteem are more likely to use downward comparisons with the motive of self-protection (12). One could speculate that patients with $\mathrm{BN}$, despite short-term reductions in body dissatisfaction (and hence, self-esteem), aim at long-term selfimprovement by orienting toward more attractive others. This would explain why they tend to set unachievably high goals in terms of weight loss. It has to be noted, however, that not only the desire for weight loss motivates dieting and disordered eating in $\mathrm{BN}$, but also other factors like the desire to gain control over the own body.

An alternative explanation of why patients with $\mathrm{BN}$ favor disadvantageous upward social comparisons is offered by the information-processing theory of body image (29). Accordingly, patients with eating disorders have easily accessible, negative self-schemata with respect to their body image, which guide information processing toward schema-consistent information. Upward social comparisons-confirming the eating disorder patients' own inferiority — would be consistent with a negative schema of the self. In line with this, submissive behaviors have been reported for patients with eating disorders (30).

The current findings have implication for research on the mass media's effects on body dissatisfaction and eating disorder symptomatology. It is likely that social comparison strategies and other processes play important roles in linking media consumption to body dissatisfaction. In our study, patients with BN focused their attention on attractive others and probably compared themselves with them, even though they had the choice not to do so, and this was accompanied by a drop in body image satisfaction. This critical role of social comparison is supported by a recent study series by Trampe, Stapel, and Siero (9) showing that body-dissatisfied individuals make more disadvantageous comparisons by comparing themselves not only with relevant peers but also with professional models, whereas body-satisfied women only compare themselves with peers. Also, the results of Tiggemann and McGill suggested that the impact of media content on body satisfaction is mediated by the level of social comparison (8). However, the reverse direction seems to work as well, as shown in Study 5 of the experiment series by Trampe and colleagues (9). When body dissatisfaction was induced experimentally in healthy individuals, they made more disadvantageous comparisons. This line of thinking is relevant to the issue of self-esteem. Following this direction of causation, low self-esteem can trigger disadvantageous comparisons, chal- lenging the notion that low self-esteem should activate the motive of self-protection, which can be accomplished by downward comparisons (12). It has to be noted here that more recent conceptualizations of social comparisons indicate that current mood influences the direction of social comparisons. According to the affect-cognition priming model (31), unhappy people make upward comparisons because negative affect makes mood-congruent comparisons more accessible (32). Thus, it is conceivable that the drop in body satisfaction in $\mathrm{BN}$ was accompanied by negative mood, thereby triggering upward social comparisons. More research is needed here and both longitudinal and experimental designs are necessary to establish the direction of causality between social comparison, mood, body dissatisfaction, self-esteem, and eating disorder symptoms.

Some limitations and methodological consideration should be noted, which are primarily related to our stimulus materials comprising a compound picture of the own body (in the center) and the two comparison bodies with higher and lower BMIs to its right and left side. This was done to assure that both comparison bodies compete for the perceiver's attention, which is probably similar to the everyday social environment, frequently comprising several potential comparison targets at a time. The inclusion of the self-picture further strongly prompted a comparison of those bodies with the self-picture and therefore most likely induced social comparison processes. However, this simultaneous presentation also brought about some limitations. First, upward and downward comparisons were necessarily inversely related (i.e., more viewing time spent on one side of the picture reduces the available time for the opposite side). An independent assessment of both upward and downward comparisons would require separate slides with higher or lower BMI bodies contrasted against the participants' own body.

As a second consequence of the use of compound pictures, attentional processes regarding the self-picture cannot clearly be separated from attention for the lateral comparison picture. Our procedure (involving initial fixations on the center and gaze shifts over the center) and calculation of the fixation time were not tailored to answer this question. Thus, the current finding that fixation times on the self-picture did not differ between groups should not be taken as evidence that biases regarding the own body do not exist in $\mathrm{BN}$. Using different procedures, specific attentional biases have been found in BN (Blechert, Ansorge, Tuschen-Caffier, submitted) and individuals with bulimic symptoms (18).

A third limitation results from the fact that we did not include neutral material. It is currently not entirely clear under what circumstances eye gaze is drawn toward negatively evaluated or positively evaluated body-related stimuli and evidence for both patterns has been presented (18, Blechert, Ansorge, Tuschen-Caffier, submitted, 33). This problem is typically circumvented by including neutral material in the procedure. Without neutral stimuli and with positive and negative material in one picture, it is not clear whether attention is drawn toward the positive material, away from the negative 
material, or a combination of both. Thus, it is also conceivable that the comparison bias described here (toward the positively evaluated information) could be a result of weight phobia (i.e., away from negatively evaluated information), which is characteristic for anorexia nervosa and has sometimes been described for healthy individuals with eating disorder symptoms (34-36).

Finally, unlike previous studies, we did not explicitly instruct participants to compare themselves with the comparison bodies but inferred comparison processes from visual attention allocation, thereby avoiding demand characteristics. Although the stimuli strongly prompted social comparisons and the Physical Appearance Comparison Scale scores testified a habitual tendency of patients with $\mathrm{BN}$ to do so, we cannot be entirely sure whether social comparison took place during picture viewing or whether other processes occurred which brought about changes in body dissatisfaction during picture viewing. Future studies comparing the viewing of body pictures under natural, uninstructed conditions and after applying an unobtrusive social comparison instruction (8) could clarify this issue. Future studies should also assess chronicity and duration of $\mathrm{BN}$ and relate this information to social comparison strategies.

With these limitations in mind, the following conclusions can be drawn. The tendency of individuals with $\mathrm{BN}$ to engage in disadvantageous social comparisons might be related to body dissatisfaction and is therefore a candidate maintenance factor. Psychotherapeutical interventions aiming at an amelioration of body dissatisfaction should therefore target more explicitly dysfunctional social comparison processes.

\section{REFERENCES}

1. Stice E, Shaw HE. Role of body dissatisfaction in the onset and maintenance of eating pathology: a synthesis of research findings. J Psychosom Res 2002;53:985-93.

2. Thompson JK, Heinberg LJ, Altabe M, Tantleff-Dunn S. Exacting beauty: theory, assessment, and treatment of body image disturbance. Washington, DC: American Psychological Association; 1999.

3. Stice E, Presnell K, Spangler D. Risk factors for binge eating onset in adolescent girls: a 2-year prospective investigation. Health Psychol 2002; 21:131-8.

4. Cash TF, Henry PE. Women's body images: the results of a national survey in the U.S.A. Sex Roles 1995;33:19-28.

5. Goldfein JA, Walsh BT, Midlarsky E. Influence of shape and weight on self-evaluation in bulimia nervosa. Int J Eat Disord 2000;27:435-45.

6. Groesz LM, Levine MP, Murnen SK. The effect of experimental presentation of thin media images on body satisfaction: a meta-analytic review. Int J Eat Disord 2002;31:1-16.

7. Tiggemann M, Slater A. Thin ideals in music television: a source of social comparison and body dissatisfaction. Int J Eat Disord 2004;35: 48-58.

8. Tiggemann M, McGill B. The role of social comparison in the effect of magazine advertisements on women's mood and body dissatisfaction. J Soc Clin Psychol 2004;23:23-44.

9. Trampe D, Stapel DA, Siero FW. On models and vases: body dissatisfaction and proneness to social comparison effects. J Pers Soc Psychol 2007;92:106-18

10. Coring AF, Krumm AJ, Smitham LA. The name assigned to the document by the author. This field may also contain sub-titles, series names, and report numbers. Differential social comparison processes in women with and without eating disorder symptoms. J Couns Psychol 2006;53:338-49.

11. Stormer SM, Thompson JK. Explanations of body image disturbance: a test of maturational status, negative verbal commentary, social comparison, and sociocultural hypotheses. Int J Eat Disord 1996;19:193-202.
12. Wood JV, Giordano-Beech M, Taylor KL, Michela JL, Gaus V. Strategies of social comparison among people with low self-esteem: selfprotection and self-enhancement. J Pers Soc Psychol 1994;67:713-31.

13. Buunk BP, Mussweiler T. New directions in social comparison research. Eur J Soc Psychol 2001;31:467-75.

14. Festinger L. A theory of social comparison processes. Human Relations 1954;7:117-40.

15. Cash TF, Cash DW, Butters JW. "Mirror, mirror, on the wall ...?" Contrast effects and self-evaluations of physical attractiveness. J Pers Soc Psychol 1983;9:351-8.

16. Klein RM. On the control of visual orienting. In: Posner MI, editor Cognitive Neuroscience of Attention. New York/London: Guilford Press; 2004.

17. Findlay JM, Gilchrist OD. Eye guidance and visual search. In: Underwood G, editor. Eye Guidance in Reading and Scene Perception. Oxford, UK: Elsevier; 1998.

18. Jansen A, Nederkoorn C, Mulkens S. Selective visual attention for ugly and beautiful body parts in eating disorders. Behav Res Ther 2005;43: 183-96.

19. Hilbert A, Tuschen-Caffier B, Ohms M. Eating Disorder Examination: Deutschsprachige Version des strukturierten Essstorungsinterviews [Eating disorders examination: a German version of the structured eating disorder interviews]. Diagnostica 2004;50:98-106.

20. Wittchen HU, Zaudig M, Fydrich T. Strukturiertes Klinisches Interview für DSM-IV. Achse I und II. Göttingen: Hogrefe; 1997.

21. Hilbert A, Tuschen-Caffier B, Karwautz A, Niederhofer H, Munsch S. Eating disorder examination-questionnaire: evaluation der deutschsprachigen Übersetzung. [Eating disorder examination-questionnaire: psychometric properties of the German version.]. Diagnostica 2007;53: $144-54$.

22. Legenbauer T, Vocks S, Schuett-Stroemel. Validierung einer deutschsprachigen Version des Body Image Avoidance Questionnaire BIAQ [Validation of the German version of the body image avoidance questionnaire (BIAQ)]. Diagnostica 2007;53:218-25.

23. Reas DL, Whisenhunt BL, Netemeyer R, Williamson DA. Development of the body checking questionnaire: a self-report measure of body checking behaviors. Int J Eat Disord 2002;31:324-33.

24. Thompson JK, Heinberg LJ, Tantleff-Dunn S. The physical appearance comparison scale. Behavior Therapist 1999;14:174

25. Ferring D, Filipp S-H. Messung des Selbstwertgefühls: Befunde zu Reliabilität, Validität und Stabilität der Rosenberg-Skala [Measurement of self-esteem: findings on reliability, validity, and stability of the Rosenberg scale]. Diagnostica 1996;42:284-92.

26. Cash TF, Fleming EC, Alindogan J, Steadman L, Whitehead A. Beyond body image as a trait: the development and validation of the body image states scale. Eating Disord 2002;10:103-13.

27. Vocks S, Legenbauer T, Heil A. Food intake affects state body image: impact of restrained eating patterns and concerns about eating, weight and shape. Appetite 2007;49:467-75.

28. Jamieson J. Analysis of covariance (ANCOVA) with difference scores. Int J Psychophysiol 2004;52:277-83.

29. Williamson DA, Stewart TM, White MA, York-Crowe E. An information-processing perspective on body image. In: Cash TF, Pruzinsky T, editors. Body Image: A Handbook of Theory, Research and Clinical Practice. New York: Guilford; 2002.

30. Troop NA, Allan S, Treasure JL, Katzman M. Social comparison and submissive behaviour in eating disorder patients. Psychol Psychother 2003;76:237-49.

31. Wheeler L, Miyake K. Social Comparison in everyday life. J Pers Soc Psychol 1992;62:760-73.

32. Wood JV, Michela JL, Giordano C. Downward comparison in everyday life: reconciling self-enhancement models with the mood-cognition priming model. J Pers Soc Psychol 2000;79:563-79.

33. Shimojo S, Simion C, Shimojo E, Scheier C. Gaze bias both reflects and influences preference. Nat Neurosci 2003;6:1317-22.

34. Hsu LK, Lee S. Is weight phobia always necessary for a diagnosis of anorexia nervosa? Am J Psychiatry 1993;150:1466-71.

35. Green MW, Elliman NA, Rogers PJ, Welch DA. Impaired color naming of food and body shape words: weight phobia or distinct affective state? Int J Eat Disord 1997;21:77-82.

36. Habermas T. In defense of weight phobia as the central organizing motive in anorexia nervosa: historical and cultural arguments for a culturesensitive psychological conception. Int J Eat Disord 1996;19:317-34. 